\title{
68 RAPID DETECTION OF SOMATIC VARIANTS IN HUMAN LEUKOCYTE ANTIGEN CLASS 1 GENES FROM SOLID TUMOR SAMPLES
}

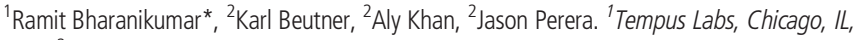
USA; ${ }^{2}$ Tempus, Chicago, IL, USA

Background Human Leukocyte Antigens (HLA) class 1 proteins are important for recognizing tumor specific mutations (neoantigens) and presenting them to CD8 + T-cells. Somatic mutations in HLA genes can potentially reduce the set of neoantigens available for presentation to T-cells, providing a possible immune escape mechanism for tumors. The presence of mutations in HLA genes may thus affect an individual's response to Immune Checkpoint Blockade therapy but calling somatic variants in HLA genes is challenging given high degrees of polymorphism and the presence of pseudogenes in HLA loci. Here, we present a rapid, modular algorithm to detect somatic variants in HLA class 1 genes from next-generation sequencing data.

Methods Our method takes as input matched tumor and normal sequencing (BAM) files and a patient's HLA type. Our first step involves mapping and aligning reads to the fulllength HLA class 1 genes of an individual patient. Next, we filter reads to minimize sequencing errors and process them to account for potential cross-mapping between genes in the broader HLA locus. Our algorithm uses the final set of processed reads from matched tumor and normal sequencing files to call somatic variants. The final set of variants are then provided with a genetic variant annotation that summarizes their functional effect.

Results We validated the efficacy of our approach using TCGA samples that were previously known to contain somatic HLA mutations. Out of a total of 46 mutations previously characterized from 41 samples, our algorithm detected 43 mutations with the correct functional annotation and did so with a computational run-time that is an order of magnitude faster than the current gold-standard approach. Eight of these mutations were previously validated using PacBio long-read sequencing, and all of these validated mutations were detected by our method. Additionally, we analyzed transcriptomic data in samples containing nonsense mutations and detected a transcriptomic signature in RNA-seq data that corresponded to the Nonsense Mediated Decay pathway.

Conclusions Our findings show that our algorithm can reliably and rapidly detect somatic mutations in HLA class 1 classical genes. We leveraged multi-modal TCGA data and modules developed at Tempus to link samples with nonsense mutations to the nonsense mediated decay pathway. This allowed us to posit a possible biological mechanism that could result in increased resistance to immunotherapy because of somatic HLA mutations. Finally, we emphasize that our approach is modular and can be extended to call somatic variants in other non-classical and class 2 HLA genes.

http://dx.doi.org/10.1136/jitc-2021-SITC2021.068 